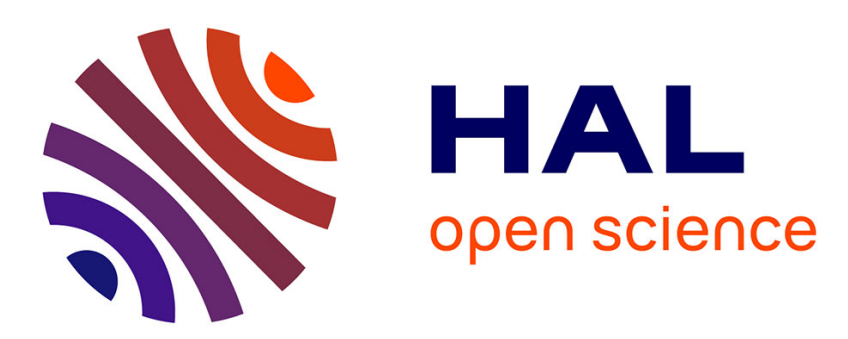

\title{
Tensor deconvolution: A method to locate equivalent sources from full tensor gravity data
}

Valentin Mikhailov, Gwendoline Pajot, Michel Diament, Antony Price

\section{To cite this version:}

Valentin Mikhailov, Gwendoline Pajot, Michel Diament, Antony Price. Tensor deconvolution: A method to locate equivalent sources from full tensor gravity data. Geophysics, 2007, 72 (5), pp.I61I69. 10.1190/1.2749317 . insu-01355054

\section{HAL Id: insu-01355054 https://hal-insu.archives-ouvertes.fr/insu-01355054}

Submitted on 22 Aug 2016

HAL is a multi-disciplinary open access archive for the deposit and dissemination of scientific research documents, whether they are published or not. The documents may come from teaching and research institutions in France or abroad, or from public or private research centers.
L'archive ouverte pluridisciplinaire HAL, est destinée au dépôt et à la diffusion de documents scientifiques de niveau recherche, publiés ou non, émanant des établissements d'enseignement et de recherche français ou étrangers, des laboratoires publics ou privés. 


\title{
Tensor deconvolution: A method to locate equivalent sources from full tensor gravity data
}

\author{
Valentin Mikhailov ${ }^{1}$, Gwendoline Pajot ${ }^{2}$, Michel Diament $^{2}$, and Antony Price ${ }^{3}$
}

\begin{abstract}
We present a method dedicated to the interpretation of full tensor (gravity) gradiometry (FTG) data called tensor deconvolution. It is especially designed to benefit from the simultaneous use of all the FTG components and of the gravity field. In particular, it uses tensor scalar invariants as a basis for source location. The invariant expressions involve all of the independent components of the tensor. This method is a tensor analog of Euler deconvolution, but has the following advantages compared to the conventional Euler deconvolution method: (1) It provides a solution at every observation point, without the use of a sliding window. (2) It determines the structural index automatically; as a consequence, the structural index follows the variations of the field morphology. (3) It uses all components of the measured full gradient tensor and gravity field, thus reducing errors caused by random noise. It is based on scalar invariants that are by nature insensitive to the orientation of the measuring device. We tested our method on both noise-free and noise-contaminated data. These tests show that tensor solutions cluster in the vicinity of the center of causative bodies, whereas Euler solutions better outline their edges. Hence, these methods should be combined for improved contouring and depth estimation. In addition, we use a clustering method to improve the selection of solutions, which proves advantageous when data are noisy or when signals from close causative bodies interfere.
\end{abstract}

\section{INTRODUCTION}

The history of gravity gradiometry dates back to 1886 when Loránd Eötvös constructed his first torsion balance gradiometer. It was the first potential field measurement device widely used in oil exploration (e.g. Bell and Hansen, 1998; Pawlowski, 1998). The first mapping of oil-bearing anticline structure was performed in Gbely, Slovakia, in 1916 (Szabó, 1998). In the 1930s, gradiometers were replaced by gravimeters and gravity measurements became easier, faster, and cheaper. Because gravity data were more easily interpretable in the precomputer era, this method was widely used.

The development of high-performance moving-platform full tensor gradiometry (FTG) systems has led to the rebirth of gravity gradiometry. The first systems measuring all components of the gravity gradient tensor (FTG) were developed in 1970s (Jekeli, 1993; Bell et al., 1997). In the late 1980s, these instruments were, for the first time, implemented in exploration geophysics (e.g. Bell and Hansen, 1998). Recently, many examples of successful applications of FTG data in mineral exploration and oil prospecting have been reported (e.g. Pawlowski, 1998; Zhdanov et al., 2004). Gravity gradiometry applications, however, are not restricted to prospecting purposes. Indeed, the European Space Agency is planning to launch the GOCE (Gravity Field and Steady-State Ocean Circulation Explorer) satellite in late 2007 with a gradiometer onboard (ESA, 1999). Tensor data will then be used in combination with GPS tracking to improve models of the global gravity field and geoid. This shall lead to unprecedented accuracy and spatial resolution, thus allowing new regional and local geodynamical studies.

In many studies, FTG data are used to calculate the enhanced gravity field $g_{z}$, which contains shorter wavelength components in comparison to gravimetry data. This allows a more detailed mapping of subsurface structures, such as the lower boundary of salt domes (Jorgensen and Kisabeth, 2000; Routh et al., 2001). Using the enhanced second vertical derivative of the potential $U_{z z}$ calculated from FTG data, joint inversion of seismic and FTG data is also performed (e.g. O'Brien et al., 2005). Several new techniques for FTG data processing and interpretation have been recently suggested (e.g. Condi and Talwani, 1999; Jorgensen and Kisabeth, 2000; Zhang et al., 2000; Li, 2001a, b; Routh et al., 2001; Lyrio et al., 2004; Zhdanov et al., 2004; and While et al., 2006). However, theory and methods for FTG data processing and interpretation that combine all FTG components and the gravity field are still challenging. We be-

Manuscript received by the Editor July 7, 2006; revised manuscript received April 6, 2007; published online July 18, 2007.

${ }^{1}$ Institut de Physique du Globe de Paris, Paris, France and Institute of Physics of the Earth RAS, Moscow, Russia. E-mail: valentin@ ipgp.jussieu.fr.

${ }^{2}$ Institut de Physique du Globe de Paris, Paris, France. E-mail: pajot@ipgp.jussieu.fr; diament@ipgp.jussieu.fr.

${ }^{3}$ Total E\&P, Non-seismic Geophysics, Paris, France. E-mail: antony.price@ total.com.

(C) 2007 Society of Exploration Geophysicists. All rights reserved. 
lieve that new marine, airborne, and space FTG measurement techniques call for the development of new methods of data processing and interpretation. Indeed, even the transformation of FTG data into enhanced gravity leads to the loss of useful information.

In this paper, we present a method to locate equivalent sources using FTG data. It is based on the same principles as Euler deconvolution, thus we call this method tensor deconvolution. It uses tensor scalar invariants and, thus, should be robust to errors caused by imperfect orientation of the measuring device. Moreover, because it uses the complete set of components of the FTG tensor, it is resistant to random noise in the different measurement channels. Contrary to the traditional Euler deconvolution method, it allows an automated estimate of the structural index and does not require a sliding window. Moreover, although this is not the first attempt to enhance Euler deconvolution by the use of gravity gradient data, this method differs from the previously published work dedicated to this effort because it uses all the measured values simultaneously, and only these values. (For example, Zhang et al., 2000, applied Euler deconvolution to FTG data considering different lines of the FTG tensor components separately. Their approach requires the calculation of the horizontal derivatives $g_{x}$ and $g_{y}$ of the gravity potential $U$.)

After recalling the fundamentals of Euler deconvolution and developing the mathematical relationships on which the algorithm is based, we present this algorithm and apply it to synthetic examples. It appears that our method may be particularly efficient at resolving the depths of multiple sources in the presence of noise.

\section{TENSOR DECONVOLUTION}

\section{Mathematical background}

Let us first briefly recall the principle of Euler deconvolution. By definition, a real function $f$ is a homogeneous function of degree $n$ when, for any $t$, it obeys the equation,

$$
f(t x, t y, t z)=t^{n} f(x, y, z) .
$$

According to this definition, the gravity and magnetic fields caused by some simple sources are homogeneous functions of the spatial coordinates. In particular, this equation is valid (see, for example, Blakely, 1995) for gravity (and magnetic) anomalies associated with point sources and lines of sources (or, in the magnetic case, point poles and point dipoles and lines of poles and dipoles). The location of a point source $(\xi, \eta, \zeta)$ in 3D, or the location of a line source $(\xi, \zeta)$ in $2 \mathrm{D}$, can be found from the following equation (Euler equation):

$$
(x-\xi) \frac{\partial f}{\partial x}+(y-\eta) \frac{\partial f}{\partial y}+(z-\varsigma) \frac{\partial f}{\partial z}=-N(f(x, y, z)-A),
$$

where $N=-n$ is the structural index, which depends on the type of the body, and $A$ is an unknown constant level in a measured field (Thompson, 1982; Reid et al., 1990). To solve equation 2, the Euler deconvolution method uses a sliding window of data points. At least four data points are required in this window, because we are solving for four unknown parameters: $\xi, \eta, \zeta$, and $A$ (e.g., Reid et al., 1990).

Strictly speaking, line and point sources are the only causative bodies that obey the Euler equation of homogeneity. Nevertheless, Euler deconvolution can also be applied to a deep body of arbitrary shape, where the anomaly is close to that of a point source or a line of sources, with corresponding structural indices $N=2$ or $N=1$ (examples of the structural indices corresponding to different causative sources are given by Stavrev, 1997). Moreover, Euler deconvolution has proven successful for edge detection of real bodies, especially simple ones having close to vertical sides. Furthermore, several bodies may obey the Euler equation under specific conditions. For example, equation 2 is valid for a dike (vertical or inclined) or a finite step when its offset is considerably smaller than its depth (Li, 2003). When the Euler method is applied to real 3D bodies, the obtained solutions very often either trace near vertical edges of causative bodies, or point to their center of mass.

Results of Euler deconvolution are sensitive to the choice of the structural index, as well as of the size and location of the sliding windows (Fairhead et al., 1994). In practice, several structural indices are tried, and the one providing results fitting to known geological and seismic data, or having good clustering properties, is kept (for an exhaustive study of the discrimination techniques to use in Euler deconvolution methods, see Fitzgerald et al., 2004). However, errors in the estimated depth of the sources occur when the index is inappropriate, and the a priori choice of a single constant index is obviously inappropriate when multiple sources with different geometries interfere. The depth estimation can be improved using additional analytical constraints, namely the property of invariance under rotation of homogeneous functions (Mushayandebvu et al., 1999). This provides additional equations and the so-called "extended Euler deconvolution method" provides better depth estimation than traditional Euler. Nabighian and Hansen (2001) mention that additional equations permit the elimination of the structural index $N$ between pairs of equations, yielding a system of two equations at each point, which are still linear in $\xi, \eta$, and s, do not contain $N$ explicitly, but are bilinear in the field variables. Discussions on methods to estimate the structural index can be found, for example, in Slack et al. (1967), Steenland (1968), Barbosa et al. (1999), and Martelet et al. (2001). As recalled by Li (2003), most methods to determine the geometry of the source (without deducing it from geology) and, thus, to guide the choice of an adequate structural index, are based on computing derivatives, and this calculation is well known to be numerically unstable, especially in the presence of noise. On the synthetic examples below, we compare our suggested tensor deconvolution method with different versions of the Euler technique, even though the comparison of extended and conventional Euler deconvolution is beyond the scope of this paper. When applying the conventional and extended Euler method we assigned the correct structural index corresponding to synthetic sources used. We believe that in this case (contrary to realistic exploration situations where the structural index is unknown) extended Euler methods provide results close to the ones obtained by conventional Euler.

Zhang et al. (2000) adapted the extended Euler deconvolution method to gravity gradient data. This allows the use of measured rather than computed derivatives, but their method requires the calculation from $g_{z}$ of derivatives $g_{x}$ and $g_{y}$ of the gravity potential along two horizontal coordinate lines, which is also known to be numerically unstable, especially in the presence of regional long-wavelength components. We hereafter describe a method to use the gravity tensor invariants computed from measured gravity gradients. Unlike previous methods, it combines the following advantages:

- Instead of a priori choosing a constant structural index, the index, which is related to the geometry of the source, is computed at every point directly from the data. The constraint brought by the knowledge of the geometry of the source to aid its localization is 
therefore deduced from the data and suitable for large data sets where the structural index is likely to vary.

- It does not require the use of a sliding window or the computation of derivatives, and, thus may be less sensitive to numerical instabilities caused by noise.

Let us now recall some fundamentals about the gravity gradient tensor. We use a Cartesian system of coordinates $(x, y, z)$ with the $z$-axis directed downwards and the $x$-axis directed northwards. The gravity gradient tensor in the $(x, y, z)$ frame can then be written in the form,

$$
\mathbf{T}=\left|\begin{array}{lll}
U_{x x} & U_{x y} & U_{x z} \\
U_{y x} & U_{y y} & U_{y z} \\
U_{z x} & U_{z y} & U_{z z}
\end{array}\right|,
$$

where $U$ is the gravity potential, and for all pair $(\alpha, \beta)$ in $\{x, y, z\} U_{\alpha \beta}$ $=\partial^{2} U / \partial \alpha \partial \beta$. In the following text, we denote by $g_{\alpha}$ the first derivative of the gravity potential $U$ along direction $\alpha$. Traditionally, gradients $U_{\alpha \beta}$ are expressed in Eötvös unit E, with $1 \mathrm{E}=10^{-9} \mathrm{~s}^{-2}$, and $g_{\alpha}$ in $\mathrm{mGal}$, with $1 \mathrm{mGal}=10^{-5} \mathrm{~ms}^{-2}$. Because gravity is a conservative field and because of the commutability of the differential operators, the tensor is symmetric $\left(U_{\alpha \beta}=U_{\beta \alpha}\right)$ and its trace is equal to zero outside of the causative sources. Thus, in free space, the tensor has only five independent components. Current commercial gradiometers, such as the Bell Geospace FTG, provide all off-diagonal and two diagonal components of the upper triangle of the gradient tensor, the third diagonal component being calculated from the two others (While et al., 2006). The tensor is fully defined from these five measurements.

Following Pedersen and Rasmussen (1990), we now investigate the scalar invariants of the tensor. Let us consider the eigenvectors $\mathbf{v}_{\mathrm{i}}$ and the eigenvalues $\lambda_{i}$ of the tensor T. Being real and symmetric, tensor $\mathbf{T}$ can be written in the form (Pedersen and Rasmussen, 1990, equation 9):

$$
\mathbf{V}^{t} \mathbf{T V}=\Lambda
$$

where $\mathbf{V}=\left[\mathbf{v}_{1}, \mathbf{v}_{2}, \mathbf{v}_{3}\right]$ is a matrix, the columns of which are eigenvectors of $\mathbf{T}$, and $\boldsymbol{\Lambda}$ is a diagonal matrix containing the three eigenvalues of the tensor. The superscript $t$ denotes the transposition of tensor T. Physically, with the origin of the coordinate system at the observation point, equation 4 means that one can find three principally different possible orthogonal rotations of the initial system of Cartesian coordinates $(x, y, z)$, such that in the new coordinate system all off-diagonal elements vanish. The eigenvectors $\mathbf{v}_{\mathbf{i}}$ determine the axes (known as the principal axes) of the new coordinate system. By definition, the tensor eigenvalues are the roots of the characteristic equation:

$$
\lambda^{3}-I_{0} \lambda^{2}+I_{1} \lambda-I_{2}=0
$$

where the $I_{i}$ coefficients are the scalar invariants of the tensor $\mathbf{T}$, the expressions of which involve only the tensor eigenvalues.

Pedersen and Rasmussen (1990) introduced the dimensionless invariant ratio $I$ associated with tensor $\mathbf{T}$ that we call hereafter the invariant ratio:

$$
I=-\left(I_{2} / 2\right)^{2} /\left(I_{1} / 3\right)^{3}, 0 \leq I \leq 1
$$

The invariant ratio $I$ is equal to zero when the field is invariant along some direction ( $2 \mathrm{D}$ causative source) and equal to 1 for radially symmetric fields (e.g., a point source, see below).

We now develop the main relationships that allow us to compute the coordinates of a point source and a line source using the invariant ratio and eigenvalues.

\section{Point source}

The gravity potential that is associated with a point source is $U$ $=G M / R$, where $G$ is the gravitational constant, $M$ is the mass of the point source, $R=\sqrt{(\xi-x)^{2}+(\eta-y)^{2}+(\varsigma-z)^{2}},(x, y, z)$ are the coordinates of the observation point, and $(\xi, \eta, \varsigma)$ those of the point source. We denote by $\lambda_{1}$ the maximal by absolute value eigenvalue of the tensor, and $\mathbf{v}_{1}$ the corresponding eigenvector. Following Pedersen and Rasmussen (1990) we get, with our sign convention,

$$
\lambda_{1}=2 G M / R^{3} \text { and } \mathbf{v}_{1}=(\xi-x, \eta-y, \varsigma-z) / R,
$$

where $\mathbf{v}_{1}$ is directed from the observation point towards the source. Thus, the eigenvector components assign the three directional angles to the source, but because $\mathbf{v}_{1}$ is a unit vector, they do not assign the distance to it. To find the three coordinates of the source, we can use the formula for the gravity anomaly $g_{z}$ (measured or enhanced/ calculated, see the introduction), which is equal to

$$
g_{z}=G M(s-z) / R^{3} .
$$

Thus, using equations 7 and 8 , we compute the depth to the point source:

$$
\varsigma-z=2 g_{z} / \lambda_{1},
$$

and the remaining $(\xi-x, \eta-y)$ coordinates can now be found from the components of vector $\mathbf{v}_{1}$. As a result, using all values of the full gradient tensor to compute $\lambda_{1}$ and knowing the value of the gravity anomaly in one point, it is possible to find the position of an equivalent point source.

Moreover, the eigenvector $\mathbf{v}_{1}$ determines a new Cartesian frame $\left(O_{1}, x_{1}, y_{1}, z_{1}\right)$, whose origin $O_{1}$ is at an observation point and where the $z_{1}$-direction coincides with $\mathbf{v}_{1}$. Thus at the origin $O_{1}$ (former $(x, y, z)$ point $)$ we have:

$$
\lambda_{1}=U_{z_{1} z_{1}}, \quad g_{z_{1}}=G M / R^{2},
$$

and equation 9 transforms to:

$$
\left(z_{1}-\varsigma\right) U_{z_{1} z_{1}}=-2 g_{z_{1}} .
$$

In the new coordinate system, derivatives $U_{x_{1} z_{1}}$ and $U_{y_{1} z_{1}}$ are equal to zero. Therefore, equation 11 is equivalent to the Euler equation for a point source with structural index 2 .

\section{For a line source directed along the $\mathrm{x}$-axis}

We denote by $M$ the mass of the line source per unit length. Then, the gravity potential is $U=-2 G M \ln (R)$ (Telford et al., 1990) and using the same notations as for the point source, and still following Pedersen and Rasmussen (1990), we have

$$
\lambda_{1}=2 G M / R^{2} \text { and } \mathbf{v}_{1}=(0, \eta-y, \varsigma-z) / R .
$$

Unit vector $\mathbf{v}_{1}$ is directed from the observation point to the nearest point of the line source (it is obvious that all these relationships are valid for an arbitrary orientation of the line source). As before, to find 
the coordinates of the source we use the gravity anomaly $g_{z}$ which is, for a line source, $g_{z}=2 G M(\mathrm{~s}-z) / R^{2}$, and thus we find the depth of the line source:

$$
s-z=g_{z} / \lambda_{1}
$$

Again, equation 13 is analogous to the Euler equation with the structural index equal to 1 .

\section{Extending formulas to real $3 D$ bodies}

Considering equations 11 and 13, we now suggest a general formula valid for elongated and isometric bodies, as follows:

$$
\varsigma=z+(1+I) g_{z} / \lambda_{1},
$$

or, equivalently,

$$
\boldsymbol{\varsigma}=z+N g_{z} / \lambda_{1} .
$$

Indeed, according to Pedersen and Rasmussen (1990) a point source has an invariant ratio $I=1$, which provides the structural index $N$ $=2$. For a line source, the invariant ratio $I$ is zero, thus $N=1$. Equation 14 thus links equations 11 and 13. For other sources, there is no strict analog of the Euler formula, instead we check our equation 14 numerically using fields generated by different causative sources. Because equation 14 is not the only way to relate the structural index to the invariant ratio $I$, further numerical studies are, of course, necessary. Using synthetic examples we investigated different power functions $N=1+I^{k}$, but for $k$ ranging from 1 to 10 , results appeared to be very close. Invariants of a tensor are, by definition, independent of the vector basis where the components of the tensor are expressed. Thus, we expect our method to be less sensitive than others to the
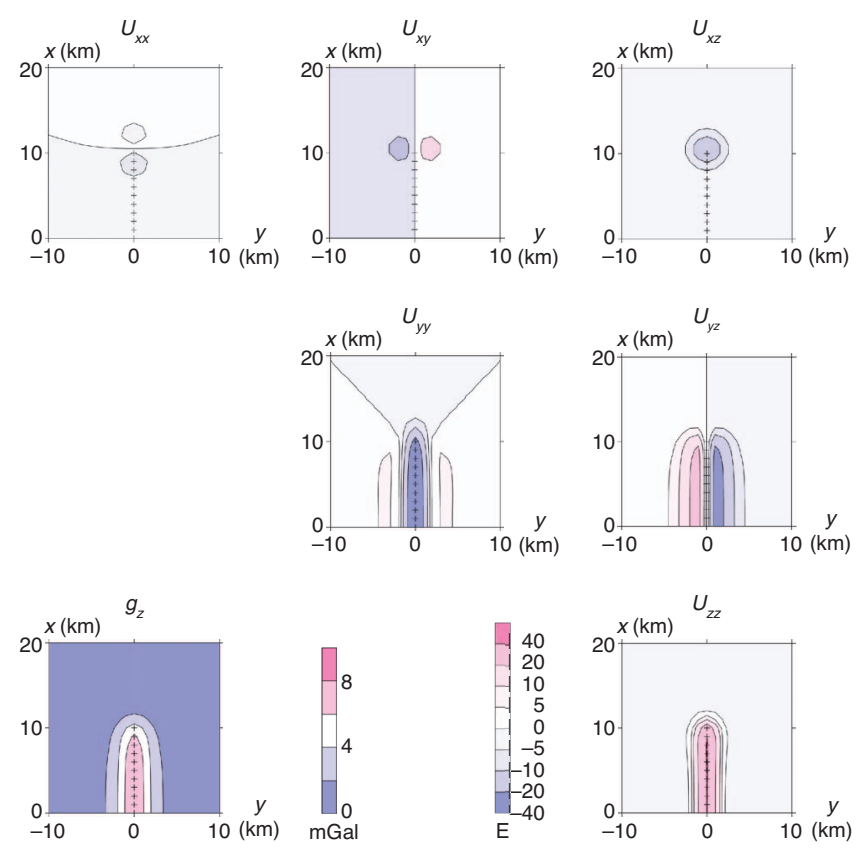

Figure 1. FTG components $U_{\alpha \beta}$ (in E) and the gravity field $g_{z}$ (in $\mathrm{mGal}$ ) caused by 21 point sources situated, one per kilometer, below the $x$-axis, between $x=-10 \mathrm{~km}$ and $x=10 \mathrm{~km}$, and all at a depth of $2 \mathrm{~km}$. Because of symmetry, only the northern part $(x \geq 0)$ of the resulting fields are shown. All distances are in kilometers. The individual effects of the point sources cannot be seen in either the gravity field or its derivatives. problems of misorientation of the measuring device. However, we do not investigate this question further in the present paper. We also do not address here the problem that some measured FTG tensor components are probably more noisy than others, as described by While et al. (2006). This would be a subject for a separate detailed investigation. We can now present the procedure for contouring causative sources and estimating their depths from FTG data.

\section{Algorithm for the tensor deconvolution}

The algorithm for the tensor deconvolution includes the following steps:

1) Calculation of eigenvalues, eigenvectors, tensor invariants, and the invariant ratio $I$ at every observation point and estimation of the structural index according to equation 14

2) Calculation of the coordinates of an equivalent source using the maximal by absolute value eigenvalue and corresponding eigenvector

3) Filtering the solutions using approaches developed for Euler deconvolution (limits along coordinates, distance from observation point to the equivalent source etc)

At step 1 we used the standard procedure suggested in Press et al. (1992). In our practical calculations, we also applied two additional approaches for the step 3 of the algorithm: solutions are rejected when their horizontal distance $L$ from the observation point is $K$ times larger than their depth $z$ ( $K$ is a user-determined parameter), and we apply clustering of the solutions as suggested by Mikhailov et al. (2003). The first criterion means that we are looking for solutions situated below the observation point within the cone whose top angle $\alpha$ is $\alpha=2 \tan ^{-1}(K)$. This criterion appeared to be very efficient. Different possible criteria to discriminate between the solutions are widely discussed by Fitzgerald et al. (2004).

\section{DISCUSSION}

In this section, we discuss the efficiency of Euler and tensor deconvolution in locating causative sources on synthetic examples. Because for isolated bodies both Euler and tensor deconvolution work well, we focus on examples of complex fields (extensive interference of signals, high noise level). For the first two examples, we show the invariants of the tensor corresponding to the investigated structures, as well as the three amplitudes of the analytic signal derivatives (see Appendix) and discuss their contouring properties. We then compare the efficiency of different versions of the Euler deconvolution method and of our method to locate the causative sources. The last example shows the ability of the algorithm to distinguish interfering 3D sources which do not obey the Euler equation.

\section{Example 1: Line of point sources}

We consider the gravity anomaly caused by 21 point sources situated, one per kilometer, below the $x$-axis, between $x=-10 \mathrm{~km}$ and $x=10 \mathrm{~km}$, and all at a depth of $2 \mathrm{~km}$. On the figures illustrating this example, only the $x>0$ part of the plane is shown, because the gravity field is symmetrical with respect to the $y$-axis.

Figure 1 shows the components of the gradient tensor and the gravity anomaly $g_{z}$ caused by these point sources. Figure 2 shows the amplitudes of the analytic signal derivatives $A_{x}$ (Figure 2a), $A_{y}$ (Figure $2 \mathrm{~b}$ ) and $A_{z}$ (Figure 2c), the first (Figure 2d) and second (Figure 
2e) invariants of the tensor, and the invariant ratio (Figure 2f). Figure 3 shows the results of our method without (Figure 3a) and with (Figure $3 \mathrm{c}$ ) clustering, those of traditional Euler deconvolution with the clustering selection criterion (Figure $3 \mathrm{~b}$ ), window size $3 \times 3 \mathrm{~km}$, and a constant structural index $N=1$ corresponding to a linear source. Isolines of $g_{z}$ are plotted in the backgrounds of Figure 3a-c.

The isolines of $A_{y}$ (Figure 2b) and $A_{z}$ (Figure 2c), as well as the first invariant (Figure 2d) contour the set of the causative sources (these functions are similar because the line of monopoles stretches along the $x$-axis). Analytic signal $A_{x}$ (Figure 2a) and the second invariant (Figure 2e) are maximal over the edge of the line of sources. The invariant ratio $I$ (Figure 2f) is close to 0 above the line of sources, and close to 1 far from it. Thus the structural index $N=I+1 \mathrm{var}-$ ies over the area. Being calculated with this varying structural index, the tensor solutions from equation 14 (Figure 3a) cluster more densely than the conventional Euler solutions computed with the constant a priori structural index $N=1$ (Figure 3b). Moreover, the tensor solutions are located in a narrower depth range than the conventional Euler ones. Thus, we conclude that in this example, our method better localizes the sources than the conventional Euler deconvolution method.

If, in addition, we use a clustering selection criterion of the tensor solutions (Figure 3c), we can even isolate all point sources, but the outermost solutions are slightly shifted toward smaller $x$ (northing) values, in comparison to the corresponding point sources. This is a surprising result considering that the depth of the sources is twice the distance between them. However, this result is achieved in absence of any kind of noise.

\section{Example 2: Noise sensitivity}

In this example, we investigate a field corresponding to a rectangular prism. This structure is far from geologically realistic, but has the advantage of being a 3D isometric body that does not obey the Euler equation. This example allows testing of equation 14. To apply
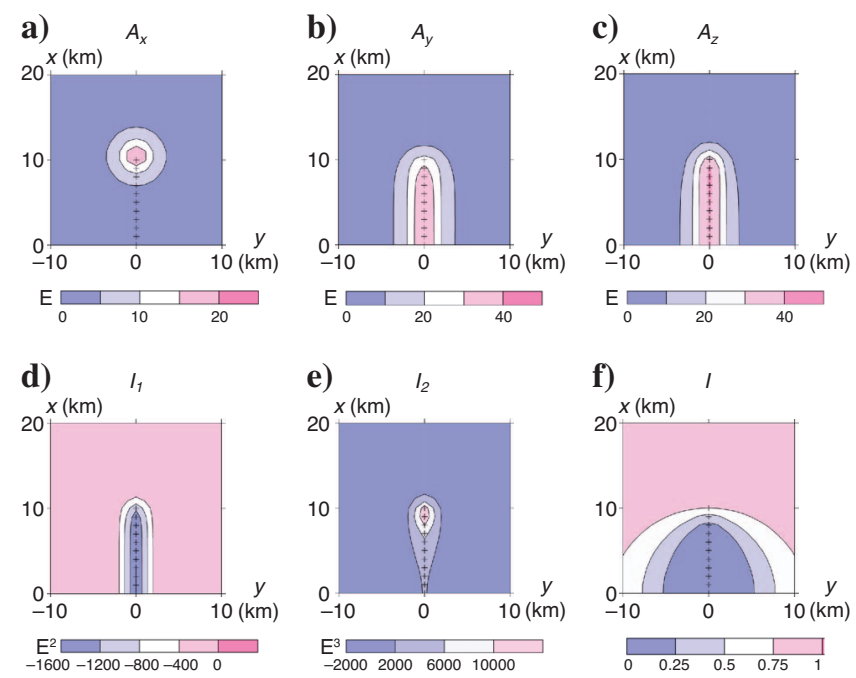

Figure 2. Amplitude of the analytic signal derivatives, invariants of the gravity gradient tensor, and invariant ratio $I$ for the example shown on Figure 1. (a), (b), and (c) The amplitude of the analytic signals derivative $A_{x}, A_{y}, A_{z}$ in Eötvös units; (d) and (e) show the first and second nonzero invariants (in $\mathrm{E}^{2}$ for $I_{1}$ and $\mathrm{E}^{3}$ for $I_{2}$ ); and (f) shows the dimensionless invariant ratio $I$. Notice the selective sensitivity of these various transforms. the conventional Euler deconvolution method, we need to assess the structural index corresponding to a prism. Zhang et al. (2000) mentions that before substitution of integral limits, the gravity field of a rectangular block resembles a homogeneous function with the structural index $N=-1$. However, the full formula with integer limits does not obey the Euler equation. Moreover, a negative structural index does not fit any potential function. Indeed, an index $N=-1$ corresponds to a function growing toward infinity.

Because at large distances the gravity effect of a rectangular prism is close to that of a point mass, its structural index approaches $N=2$ as the distance tends to infinity. At shorter distances the structural index $N=1$ corresponding to a small-amplitude step can be used. (Note that this supports the idea of an effective structural index changing with the distance from a source). For this example, we choose to apply the conventional and extended Euler deconvolution method with a constant a priori structural index $N=2$.

The gravity field and its derivatives are calculated for a rectangular body of $10 \times 10 \mathrm{~km}$ horizontal dimensions, stretching down from 2 to $30 \mathrm{~km}$ and having a density contrast equal to $1 \mathrm{~g} / \mathrm{cm}^{3}$.

Figure 4 shows the tensor components and $g_{z}$ associated with this structure. Figure 5 shows the amplitudes of the analytic signal derivatives $A_{x}$ (Figure 5a), $A_{y}$ (Figure 5b), and $A_{z}$ (Figure 5c), the first (Figure $5 \mathrm{~d}$ ) and second (Figure 5e) invariants of the tensor, and the invariant ratio (Figure 5f). Figure $5 \mathrm{a}$ and $\mathrm{b}$ demonstrate the selective directional sensitivity of the $A_{x}$ and $A_{y}$ components, which allows
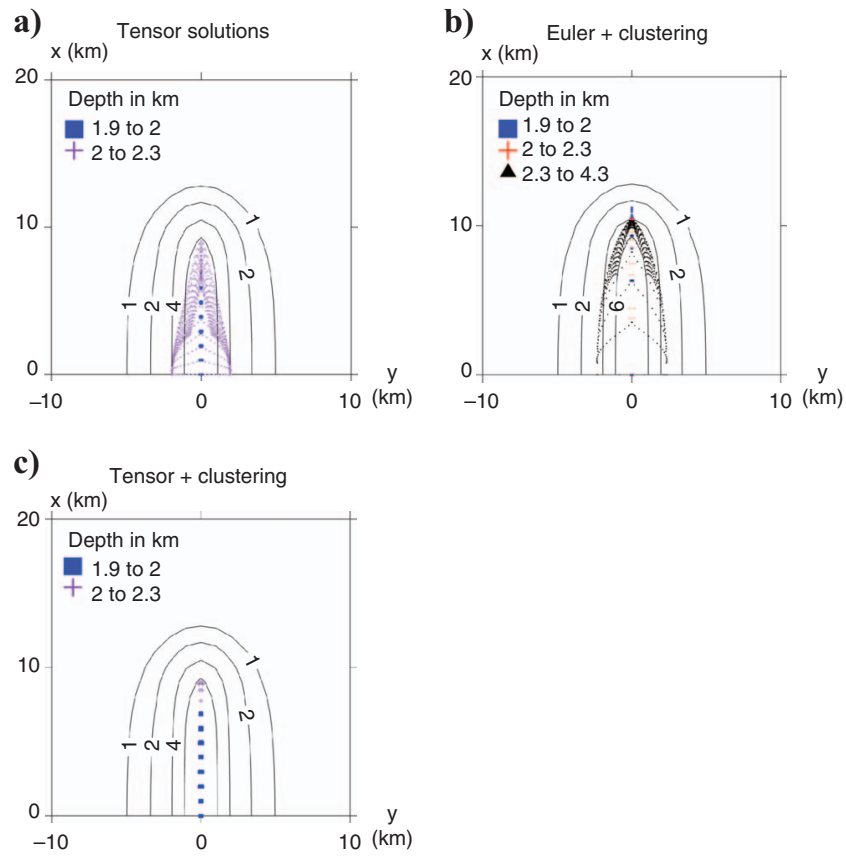

Figure 3. Comparison of tensor and Euler deconvolution for the example illustrated by Figures 1 and 2. (a) The results of tensor deconvolution, (b) the Euler solutions after selection and clustering, and (c) the tensor solutions selected by clustering. All figures are with the gravity field $g_{z}$ in the background. Blue squares, red crosses, and black triangles indicate different depth intervals in kilometers. Notice that Euler solutions (b) form a wider cloud than the tensor solutions (a). Tensor solutions (a) are located close to the sources. After the clustering of the tensor solutions (c) all point sources are recognized, though the outermost solutions are shifted to smaller $x$ values with respect to the corresponding point sources. The depths of the solutions are then very close to the real depth of $2 \mathrm{~km}$. 
improved outlines of the different edges of the causative bodies. We can also notice the variation of the invariant ratio $I$ above the vertical sides of the prism (Figure 5f).

First we add Gaussian random noise with zero average and standard deviations of $1 \mathrm{mGal}$ and $1 \mathrm{E}$ to the gravity field and to all FTG
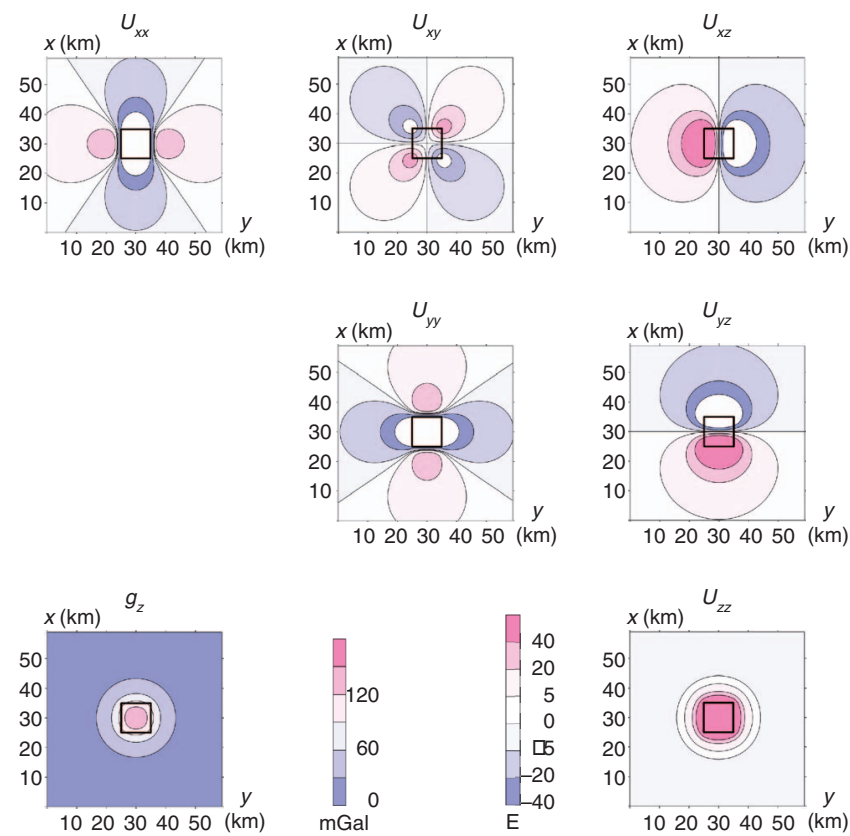

Figure 4. FTG components $U_{\alpha \beta}$ (in E) and gravity field $g_{z}$ (in mGal) caused by a rectangular body of horizontal dimensions $10 \times 10 \mathrm{~km}$. The top is at $2 \mathrm{~km}$ and the bottom at $30 \mathrm{~km}$, excess density is $1 \mathrm{~g} / \mathrm{cm}^{3}$. The solid square on the plots shows the contour of the causative body.
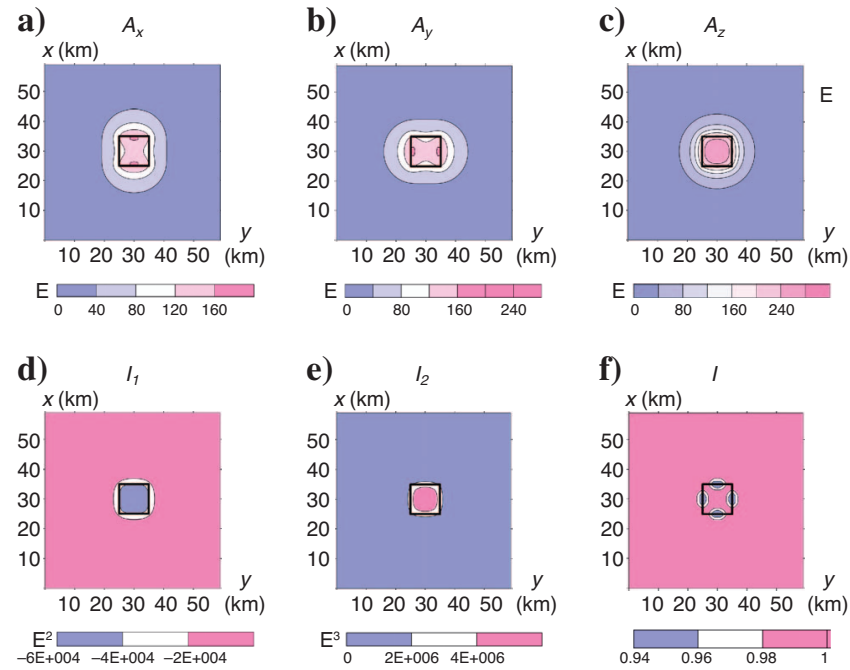

Figure 5. Same as Figure 2, except for the example shown on Figure 4. The solid square shows the contour of the causative body. Notice that the transform $A_{x}$ outlines the boundaries that are perpendicular to the $x$-axis and $A_{y}$ the ones perpendicular to the $y$-axis. Because the gravity field is isometric, the behavior of the invariants is similar to that of transform $A_{z}$. As a result, the invariant ratio $I$ is everywhere close to 1 . components, respectively (Figure 6). Then we investigate the effect of noise with larger standard deviations, $3 \mathrm{mGal}$ and $7 \mathrm{E}$, respectively (Figure 7).

Figure 6 shows the results of our method (Figure 6a) and of conventional Euler deconvolution (Figure $6 \mathrm{~b}$ ) with window size $3 \times 3 \mathrm{~km}$. For the tensor deconvolution, solutions were selected using two criteria:

1) Solutions are required to have positive depth

2) Solutions whose horizontal distance from the observation point is $K$-times larger than their depth are rejected (we used $K=1$, thus looking for solutions situated below the observation point, within the cone with top angle $90^{\circ}$ )

For the low-noise example, we restricted the conventional Euler deconvolution method, used as a comparison, by applying both routine selection and clustering. This was necessary because Euler solutions were more widely dispersed.

Figure $6 \mathrm{a}$ and $\mathrm{b}$ demonstrate that our method better locates the center of the anomalous body, whereas conventional Euler solutions better identify its edges. This suggests that these methods are complementary and can be applied simultaneously to better locate causative bodies. The tensor solutions are, however, better at determining a more accurate depth for the causative body modeled here.

When the level of noise increases (Figure 7a-c) the depth accuracy of the conventional Euler solutions increases drastically, and the edges of the body are not well outlined (Figure 7b). Though almost the same selection criterion are applied in the cases shown on Figures $6 \mathrm{a}$ and $7 \mathrm{a}$, the tensor solutions remain very densely clustered in the center of the body, being distributed in a narrow depth range. To better outline the causative source by conventional Euler and clustering, we applied stronger selection criteria, thus considerably reducing the number of solutions (Figure $7 b$ ). Very strong selection criteria applied to extended Euler deconvolution (LCT software, structural index 2 , and window size $36 \times 36$ points) result in a very dense deep cluster situated within a narrow depth interval (Figure
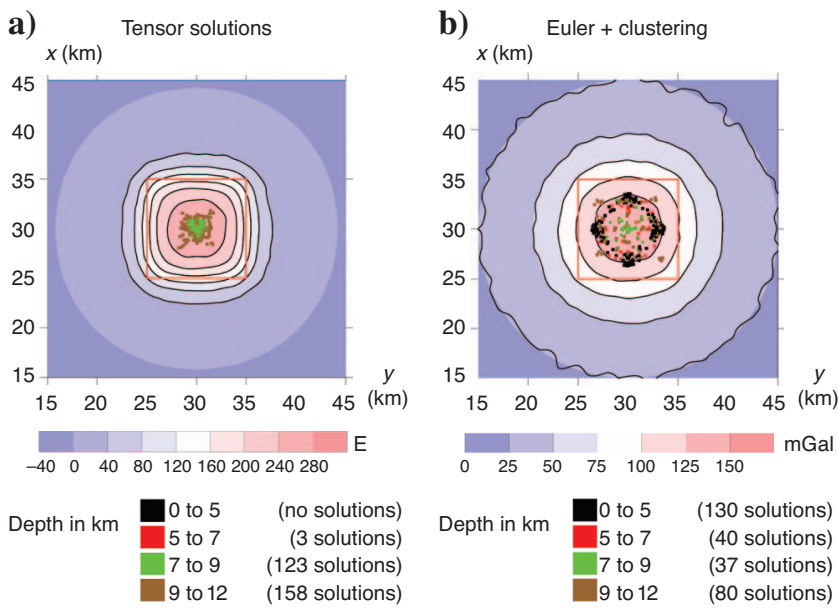

Figure 6. Results of tensor (a) and Euler (b) deconvolution of data in the presence of noise. A random Gaussian noise with zero average and standard deviation of $1 \mathrm{mGal}$ and $1 \mathrm{E}$ was added to the gravity field and all FTG components. Color scales show fields without noise $\left[U_{z z}\right.$ on (a) and $g_{z}$ on (b)], whereas isolines show the noisy fields. Colored symbols show the depth intervals for the solutions. See the text for more details. 
7c). This example indicates that our method is robust to the noncovariant Gaussian noise, even when applied to structures that do not obey the Euler equation. In this paper, we do not address the question of a possible covariance of noise in the FTG components.

\section{Example 3: Combination of interfering sources}

We now show on Figure 8 a synthetic example involving three bodies:

1) A thin dike at the top of the figure with its top at $0.5 \mathrm{~km}$ depth

2) A rectangular block (bottom right) with its top at $1 \mathrm{~km}$

3) A rectangular block (bottom left) with its top at $2 \mathrm{~km}$

The lower boundaries of the three bodies are at 10-km depth. The excess density of the dike is two times larger than that of the other blocks, but its total mass is nearly three times smaller, so the anomaly (image background) of the dike is considerably less prominent than those of rectangular bodies. We can notice the coalescence of anomalies from the rectangular blocks at the bottom.

a)

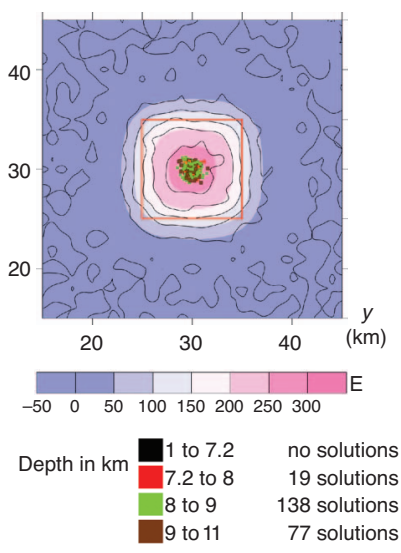

b)

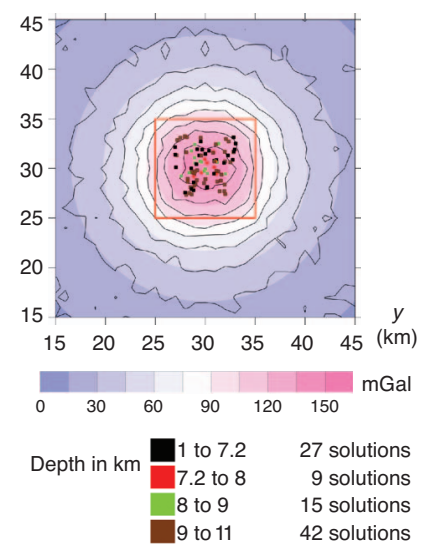

c)

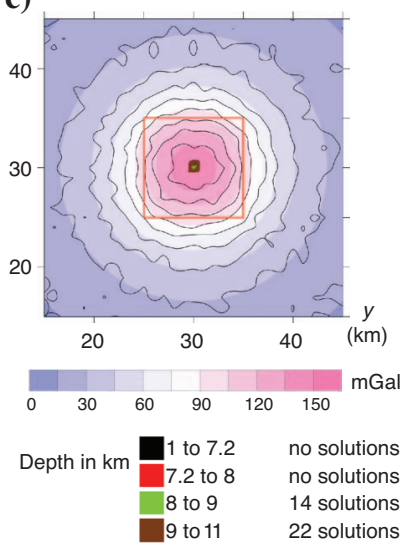

Figure 7. The same as Figure 6, but with a higher level of noise (random Gaussian noise with zero average and standard deviation of $3 \mathrm{mGal}$ and $7 \mathrm{E}$ ). (a) Tensor solutions 234 and (b) Conventional Euler solutions 93 after clustering. (c) The results of extended Euler deconvolution after strong selection (only 36 solutions left). The depth interval of the solutions is smaller than on Figure 6 because of the strong selection.
Figure $8 \mathrm{a}$ and $\mathrm{b}$ show the results of the tensor deconvolution method, with the two following selection criteria:

1) Figure 8a: rejecting solutions whose distances from the observation point are larger than twice their depth $\left(\alpha=26^{\circ}\right)$

2) Figure 8b: rejecting solutions whose distances from the observation point are larger than their depth $\left(\alpha=90^{\circ}\right)$. For this example, the clustering of the solutions method was also applied

Figure $8 \mathrm{c}$ and $\mathrm{d}$ show the results of the extended Euler deconvolution method, with window size $5 \mathrm{~km}$ and structural index 1 and 2, respectively. Note that the extended Euler depth estimations were performed independently, and that no a priori knowledge of the source body depth, shape, or distribution was provided.

Though different rejection criteria were used (even additional clustering for Figure $8 \mathrm{~b}$ case), the results presented on plots for Figure $8 \mathrm{a}$ and $\mathrm{b}$ are close to each other. Tensor solutions clearly show the central parts of the rectangular blocks and demonstrate that they are well separated. The thin dike at the top of the figure is also well outlined. Solutions also show that the dike is shallower than the blocks and that the block to the right is shallower than its neighbor on the left. The extended Euler solutions (Figure $8 \mathrm{c}$ and d) are more widely dispersed, showing edges of the causative bodies. The position of the thin dike at the top, as well as the separation of the two blocks at the bottom, is less clear than in the tensor solutions case. This indicates that the tensor deconvolution method may be more stable to the interference of signals from close causative bodies. a)

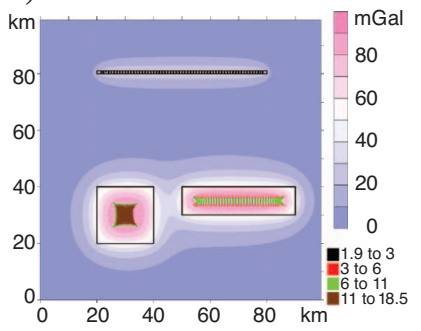

c)

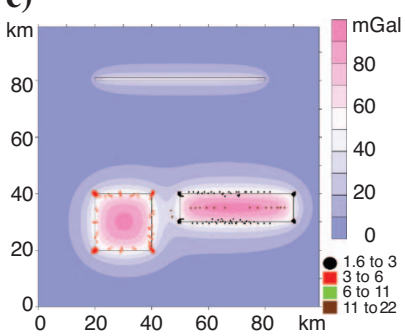

b)

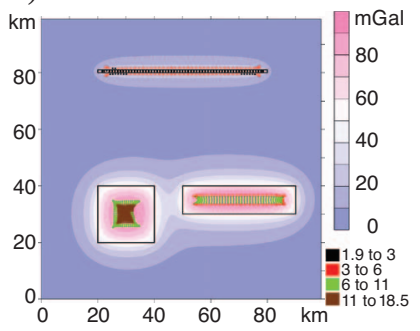

d)

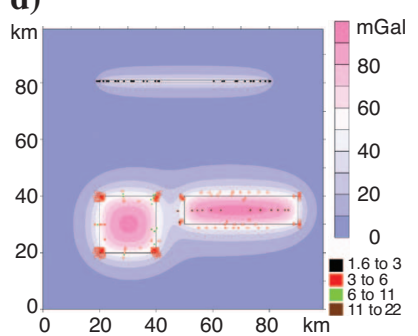

Figure 8. (a) and (b) Results of tensor deconvolution with different rejection criteria applied for the selection of the solutions; (c) and (d) extended Euler deconvolution for a combination of three sources, in the absence of noise. Square box legends with symbols indicating the depth of the solutions in kilometers are given for each plot. The colored backgrounds stand for $g_{z}$ in $\mathrm{mGal}$. Though different criteria were used for the selection of the solutions, the results of tensor deconvolution on (a) and (b) remain close to each other; (c) and (d) show solutions obtained using extended Euler deconvolution (LCT software) with a window size of $5 \times 5$ points and a structural index of 1 (c) and 2 (d). The bodies are better separated by the tensor solutions when their edges are better outlined by the traditional Euler solutions, so these methods are complementary. 


\section{CONCLUSION}

We have described here our new method to locate causative sources from gravity gradiometry data. As it is analogous to the Euler deconvolution method and uses all full tensor gravity gradient components, we call it tensor deconvolution. It must be noted again that several improvements of the traditional Euler deconvolution method have been proposed, such as the extended Euler deconvolution method or Euler deconvolution of the analytic signal. Our aim was not to develop a method that improves Euler deconvolution, but to develop a method suited to the interpretation of gravity gradiometry data, and taking advantage of the complete set of components of the gravity gradient tensor measured by a gradiometer. The tensor deconvolution method is therefore complementary to the traditional Euler deconvolution method rather than its enhancement. Several differences between our approach and several routine Euler deconvolution methods must, however, be outlined:

- Tensor deconvolution provides a solution at every observation point, without using a sliding window, and thus is not sensitive to the size or the location of such a window.

- It determines the structural index automatically from the data, and as a consequence, the structural index follows the variations of the field morphology.

- It uses the gravity field and all components of the measured full gradient tensor. Because gravity gradiometry measurements are performed independently from gravity ones, and because the tensor components are considered simultaneously, errors caused by any random noise are likely to be reduced. The robustness of tensor deconvolution (compared with traditional Euler deconvolution), applied to increasingly noisy data has been demonstrated.

- The gravity field derivatives are used through the scalar invariants of the tensor. Because the invariants are by definition independent from the basis on which they are computed, the results should be insensitive to the orientation of measuring devices. Further work with real data will allow us to investigate this promising property of the invariants.

Note that even if sliding windows are not necessary, they could be useful, especially in the presence of noise. The use of sliding windows actually allows the introduction of an unknown constant in equation 14 , writing $\left(g_{z}-A\right)$ instead of $g_{z}$. The possibility of introducing such a constant is useful because real measurements provide relative values of the gravity anomaly. We do not recommend introducing independent constants for every sliding window, because in this case, one subtracts, not a constant level, but some continuous field component that changes (sometimes dramatically) from one point to another. Introducing one constant for the whole study area or for relatively large domains is therefore preferable. Moreover, if the measured field contains components with different wavelengths, we recommend prior simultaneous filtering of the gravity field and its FTG components, allowing for the fact that they are derivatives of the same potential function $U$. An equivalent sources technique may be used for this filtering.

Lastly, clusters of tensor solutions localize the center of causative bodies, whereas the Euler solutions traditionally better outline their edges. Thus, these methods should best be combined to better identify the sources and estimate their depths. Clustering of solutions, as proposed by Mikhailov et al. (2003), is indeed a powerful tool, especially useful for noisy data or if signals from various sources interfere.

\section{ACKNOWLEDGMENTS}

Our paper greatly benefited from a very extensive revision by nine reviewers. We thank Ed Biegert, Horst Holstein, Xiong Li, Laust Pedersen, Alan Reid and four anonymous reviewers for their helpful comments and suggestions that allowed us to greatly improve the original version of this manuscript. V. Mikhailov acknowledges financial support of Russian Foundation for Basic Research (grant 0605-64629). G. Pajot benefited from a DGA grant, and this study was supported by the French Space Agency CNES. This is IPGP contribution $\mathrm{N} 2225$.

\section{APPENDIX A}

\section{RELATIONSHIPS BETWEEN THE TENSOR INVARIANTS AND THE ANALYTIC SIGNAL}

According to Roest et al. (1992), the gravity analytic signal is

$$
A(x, y, z)=g_{x} \mathbf{e}_{x}+g_{y} \mathbf{e}_{y}+i g_{z} \mathbf{e}_{z},
$$

where $i$ is the complex unit, and $\left(\mathbf{e}_{x}, \mathbf{e}_{y}, \mathbf{e}_{z}\right)$ are unit vectors in directions $x, y$, and $z$ respectively. The amplitudes of the directional derivatives of the analytic signal are

$$
\begin{aligned}
& A_{x}=\sqrt{U_{x x}^{2}+U_{x y}^{2}+U_{x z}^{2}}, \\
& A_{y}=\sqrt{U_{x y}^{2}+U_{y y}^{2}+U_{y z}^{2}}, \\
& A_{z}=\sqrt{U_{x z}^{2}+U_{y z}^{2}+U_{z z}^{2}},
\end{aligned}
$$

and, thus, may be calculated using rows of the full gravity gradient tensor. Those amplitudes possess a selective sensitivity in different directions, and can be used for tracing faults or close to vertical sides of causative bodies.

Considering the expressions of the three amplitudes $A_{x}, A_{y}$, and $A_{z}$ given in equation A-2, we infer from equation $10 \mathrm{~b}$ by Pedersen and Rasmussen (1990) that the first nonzero scalar invariant can be written as

$$
I_{1}=-\left(A_{x}^{2}+A_{y}^{2}+A_{z}^{2}\right) / 2 .
$$

The synthetic examples in the text illustrate the contouring properties of the derived transforms. We give a comparative analysis of the morphology of the three amplitudes of the directional derivatives of the analytic signal and of the invariants when discussing these examples.

\section{REFERENCES}

Barbosa, V. C. F., J. B. C. Silva, and W. E. Medeiros, 1999, Stability analysis and improvement of structural index estimation in Euler deconvolution: Geophysics, 64, 48-60.

Bell, R. E., R. Anderson, and L. Pratson, 1997, Gravity gradiometry resurfaces: The Leading Edge, 16, 55-59.

Bell, R. E., and R. O. Hansen, 1998, The rise and fall of early oil field technology: The torsion balance gradiometer: The Leading Edge, 17, 81-83.

Blakely, R. J., 1995, Potential theory in gravity and magnetic applications: Cambridge University Press.

Condi, F., and M. Talwani, 1999, Resolution and efficient inversion of gravi- 
ty gradiometry: 69th Annual International Meeting, SEG, Expanded Abstracts, 358-361.

ESA, 1999, ESA gravity field and steady-state ocean circulation explorer, Reports for mission selection, the four candidate earth explorer core missions: SP-1233

Fairhead, J. D., K. J. Bennett, D. R. H. Gordon, and D. Huang, 1994, Euler: Beyond the "black box": 64th Annual International Meeting, SEG, Expanded Abstracts, 422-424

Fitzgerald, D., A. Reid, and P. McInerney, 2004, New discrimination techniques for Euler deconvolution: Computers and Geosciences, 30, 461-469.

Gordin, V. M., B. O. Mikhailov, and V. O. Mikhailov, 1980, Physical aspects of anomalous fields approximation and filtration: Izvestiya, Physics of the Solid Earth, 16, 52-61.

Jekeli, C., 1993, A review of gravity gradiometer survey system data analysis: Geophysics, 58, 508-514.

Jorgensen, G. J., and J. L. Kisabeth, 2000, Joint 3-D inversion of gravity, magnetic and tensor gravity fields for imaging salt formations in the deep water Gulf of Mexico: 70th Annual International Meeting, SEG, Expanded Abstracts, 424-426.

Keating, P., and M. Pilkington, 2004, Euler deconvolution of the analytic signal and its application to magnetic interpretation: Geophysical Prospecting, 52, 165-182.

$\mathrm{Li}, \mathrm{X} ., 2003$, On the use of different methods for estimating magnetic depth: The Leading Edge, 22, 1090-1099.

Li, Y., 2001a, Processing gravity gradiometer data using an equivalent source technique: 71st Annual International Meeting, SEG, Expanded Abstracts, $1466-1469$.

, 2001b, 3-D inversion of gravity gradiometer data: 71st Annual International Meeting, SEG, Expanded Abstracts, 1470-1473.

Lyrio, J., L. Tenorio, and Y. Li, 2004, Efficient automatic denoising of gravity gradiometry data: Geophysics, 69, 772-782.

Martelet, G., P. Sailhac, F. Moreau, and M. Diament, 2001, Characterization of geological boundaries using $1 \mathrm{D}$-wavelet transform on gravity data: Geophysics, 66, 1116.

Mikhailov, V. O., A. Galdeano, M. Diament, A. Gvishiani, S. Agayan, S. Bogoutdinov, E. Graeva, and P. Sailhac, 2003, Application of artificial intelligence for Euler solution clustering: Geophysics, 68, 168-180.

Mushayandebvu, M. F., P. van Driel, A. B. Reid, and J. D. Fairhead, 1999 Magnetic imaging using extended Euler deconvolution: 69th Annual International Meeting, SEG, Expanded Abstracts, 401-402.

Nabighian, M. N., and R. O. Hansen, 2001, Unification of Euler and Werner deconvolution in three dimensions via the generalized Hilbert transform:
Geophysics, 66, 1805-1810.

O'Brien, J., A. Rodriguez, D. Sixta, M. A. Davies, and P. Houghton, 2005 , Resolving the K-2 salt structure in the Gulf of Mexico: An integrated approach using prestack depth imaging and full tensor gravity gradiometry, The Leading Edge, 24, 404-409.

Pawlowski, B., 1998, Gravity gradiometry in resource exploration: The Leading Edge, 17, 51-52.

Pedersen, L. M., and T. M. Rasmussen, 1990, The gradient tensor of potential anomalies: Some implications on data collection and data processing of maps: Geophysics, 55, 1558-1566.

Press, W. H., S. A. Teukolsky, B. P. Flannery, and W. T. Vetterling, 1992, Numerical recipes in Fortran: the Art of Scientific Computing, 2nd ed.: Cambridge University Press.

Reid, A. B., J. M. Allsop, H. Grancer, A. J. Millett, and I. W. Somerton, 1990, Magnetic interpretation in three dimensions using Euler deconvolution Geophysics, 55, 80-91.

Roest, W. R., J. Verhoev, and M. Pilkington, 1992, Magnetic interpretation using the 3D analytic signal: Geophysics, 57, 116-125.

Routh, P., G. J. Jorgensen, and J. L. Kisabeth, 2001, Base of the salt mapping using gravity and tensor gravity data: 70th Annual International Meeting, SEG, Expanded Abstracts, 1482-1484.

Slack, H. A., V. M. Lynch, and L. Langan, 1967, The geomagnetic gradiometer: Geophysics, 32, 877-892.

Stavrev, P. Y., 1997, Euler deconvolution using differential similarity transformations of gravity or magnetic anomalies: Geophysical Prospecting, 45, 207-246.

Steenland, N. C., 1968, Discussion on: "The geomagnetic gradiometer," H. A. Slack, V. M. Lynch, and L. Langan, authors: Geophysics, 33, 681-683.

Szabó, Z.. 1998, Three fundamental papers of Loránd Eötvös: Eötvös Loránd Geophysical Institute of Hungary.

Telford, W. M., L. P. Geldart, and R. E. Sheriff, 1990, Applied Geophysics: Cambridge University Press.

Thompson, D. T., 1982, EULDPH: A new technique for making computerassisted depth estimates from magnetic data: Geophysics, 47, 31-37.

While, J., A. Jackson, D. Smit, and E. Biegert, 2006, Spectral analysis of gravity gradiometry profiles: Geophysics, 71, no. 1, J11-J22.

Zhang, C., M. F. Mushayandebvu, A. B. Reid, J. D. Fairhead, and M. Odegard, 2000, Euler deconvolution of gravity tensor gradient data: Geophysics, 65, 512-520.

Zhdanov, M. S., R. Ellis, and S. Mukherjee, 2004, Three-dimensional regularized focusing inversion of gravity gradient tensor component data: Geophysics, 69, 925-937. 